\title{
Spine Surgery Database: Spine Disease Analytical Evaluation Module
}

\author{
Semih UTKU \\ Dokuz Eylul University, Department of Computer Engineering, Izmir, Turkey \\ Corresponding author: Semih UTKU semih@cs.deu.edu.tr
}

\section{ABSTRACT}

AIM: To demonstrate a custom spine disease analytical evaluation module that can instantly, accurately, and effectively analyze the widely accepted outcome measurements.

MATERIAL and METHODS: The data input and analysis processes were evaluated on a timely basis to compare the traditional paper-based manual data entry method with the spine surgery database (SSD) platform data entry method. The data of 116 patients in the cervical degenerative patient group were analyzed using the upgraded version of the SSD. The subjects were analyzed with respect to the SF-36 Quality of Life Index, Nurick classification, and Japanese Orthopedic Association score. The manual and computerized patient information analyses were then compared.

RESULTS: The developed analysis module enables the instantaneous access and analysis of patient data. More importantly, the paperless evaluation module improves the post-surgery patient status evaluation time by $45.64 \%$. For 116 patients, a physician gains up to $401 \mathrm{~min}$ of evaluation time in each preoperative and follow-up period without being subjected to the human errors encountered in paper records.

CONCLUSION: It is apparent that customized software solutions are absolutely necessary in patient registration and follow-up processes. The experimental results showed that using the proposed module, patient follow-up and progress analyses can be performed in a fast, effective, and accurate manner.

KEYWORDS: Spine surgery, Outcome analysis, Analytical evaluation

\section{INTRODUCTION}

$\mathrm{S}$ ignificantly large amounts of data are generated in the health industry, which include the follow-up records as well as the diseases and history of patients (8). However, in many countries, these data are still being manually recorded on paper. Such manual methods are ineffective for providing appropriate and efficient solutions for healthcare (2). It is evident that there is an urgent need for fast health data storage and processing systems to increase the reliability of patient reports by transferring the data in the paper files to computers. Therefore, efforts to accelerate the digitization and computerization of all health processes need to be supported to make healthcare information open, easily sharable, and interoperable. This will in turn improve the quality of healthcare decision support and disease surveillance (11). Additionally, the available databases and hospital applications can be used to facilitate patient followup for subsequent examinations and evaluation (6). In medical processes, post-surgery improvements in patients must be closely monitored by regularly collecting patient data. Patient tracking software integrated with analytical assessment tools make a significant contribution to the physicians' decisionmaking process (10). In the Oregon Health and Science University Spine Clinic, a general survey system has been developed based on the SF-12 and Oswestry Disability Index to facilitate patient follow-up procedures. For the followup process of spine surgery patients, the questionnaires/ surveys are generally paper-based; for example, the SF-36 Quality of Life Index for evaluating the difficulty in walking,

Semih UTKU (1) : 0000-0002-8786-560X 
Nurick classification and Japanese Orthopedic Association (JOA) score for the level of disability, Oswestry Disability Index, and Visual Analog Scale for Pain (15). Furthermore, computerized automation software can be used to provide a systematic structure for rapid post-surgery decision-making. Such structures should be developed to have user-friendly interfaces and analysis tools that will enable the evaluation to be performed from different perspectives. The increased use of such tools is bound to contribute to the physician decisionmaking process, patient follow-up evaluations, and, more importantly, the transfer of information to other physicians by exemplifying the information.

\section{Problem Definition and Contribution}

The problems encountered in the follow-up surveys using existing hospital applications are as follows:

- Collection of patient questionnaires in non-electronic forms, on error prone papers

- Labor intensive effort in the evaluation of the collected questionnaires

- Lack of timely and accurate results to patients and physicians

- Lack of graphical aids to physicians in data interrogation

- Inability to evaluate the results of surveys instantaneously

- Inability to obtain cannot be monitored instantaneous statistical information out of the survey results

- Incorrect data transfer by specialists from paper to a computer environment

- Absence of specialized and integrated electronic health records for spine surgery

The most important contribution of this study is the improvement in the quality of health services through the use of our custom module that provides a tool to address the aforementioned problems. Our study aims to persuade physicians to use the Spine Surgery Database (SSD) in the widely accepted standard follow-up and treatment process questionnaires.

\section{ם MATERIAL and METHODS}

The first Turkish SSD application was developed in 2006 (12), and provides a platform for recording spine surgery patient data. Being a specialized software in the field of spine surgery, the SSD regularly stores patient data that helps in the management of detailed patient monitoring and in providing on demand and fast access to the data. Approximately 22,000 patients were registered in the SSD application in 2010; and the number of patients that are currently registered is approximately 36,000 . The SSD software was developed based on the requirements of the physicians using the tool. Recently, an analysis module was added to the SSD for analyzing the follow-up process. Thus, to the best of our knowledge, this is the first paperless environment developed for tracking patients in Turkish spine surgery clinics.
The following standards were considered in this study:

a) Nurick classification: The Nurick scale defines the early clinical symptoms of walking anomaly when a patient is examined for the first time. Hence, walking problems were classified based on the Nurick categories. The categories range from a grade of zero to five, where grade zero implies that the patients only have root symptoms, and grade five implies that the patients are chair bound or bedridden.

b) JOA score: Evaluations were performed using the modified JOA score (3). The modified JOA scale consists of four domain scores, i.e., motor dysfunction score in the upper extremities, motor dysfunction score in the lower extremities, sensory dysfunction score of the upper extremities, and sphincter dysfunction score, with the score ranging from 0 to $5,7,3$, and 3 , respectively, with the minimum total score being 0 and the maximum being 17 (16). Myelopathy is considered to be mild if the JOA score is greater than 13 , moderate if the JOA score ranges from 9 to 13, and severe if the JOA score is less than 9. Keller et al. published the modified version in German (7).

c) Thirty-Six-Item Short Form Health Survey (SF-36): SF-36 is the most widely used survey for measuring the quality of life. It consists of 36 questions related to the general health status of patients, and provides eight specific categories of physical and emotional scores, i.e., physical functioning, role limitation due to physical problems, bodily pain, general health, vitality, social functioning, role limitation due to emotional problems, and mental health (9). For each quality of life parameter, the item scores were coded, summed, and transformed into a scale from 0 (worst) to 100 (best) using the standard SF-36 scoring algorithms (5).

The scores of these three widely accepted evaluation standards were implemented into the SSD application. The entered data were then analyzed and evaluated using the SSD analytical module.

\subsection{SSD Software}

The SSD software (12) was developed as a Windows Forms application using the $\mathrm{C} \#$ programming language in the Microsoft Visual Studio environment. The use case diagram of the SSD application is illustrated in Figure 1. In addition to the general operational functions of the SSD application, a spine data analytical evaluation module extension was also added. Furthermore, a GUI feature was developed to help doctors in evaluating and analyzing the results. Thus, the doctors could monitor and manage the results of assessments (JOA, Oswestry, SF 36) in a quick and efficient manner.

\subsection{SSD Analytical Evaluation Module}

The spine data analytical evaluation module was created as a new feature for the SSD software; its visualizations/ graphical drawings were developed through the drawing library .netCHARTING 3.0 (13). The impressions made in the module can be shown instantaneously after patient data entry. In addition, the data regarding the average patient survey results for each scale in the graph are also instantaneous. Thus, the physicians can make an overall assessment of the patient's condition. The patient follow-up data entry interface 
for the analysis module is depicted in Figure 2. Physicians or their secretaries can enter the Nurick classification data, JOA score data, and SF-36 questionnaire data using the follow-up module during the patient's follow-up period. At this point, the data entered for each patient are systematically recorded in the SSD system on the relevant follow-up date. These data can be used to perform graphical instantaneous data analysis through the SSD analytical evaluation module.

Figure 3A presents an example of the SSD evaluation module interface (Nurick score results) in which the average admission and follow-up data are generated according to the entered data in the cervical degenerative disorders group. Physicians can instantaneously obtain the results and view the Nurick score, JOA score, and SF-36 results on a historical basis for a particular patient. Similarly, Figure 3B presents the interface that shows the follow-up JOA scores of a particular patient.

Figure 4 shows the SF-36 questionnaire results used for evaluating the quality of life data of patients. This assessment environment can help spine surgery specialists to analyze the changes in the survey results that are entered momentarily. The mean changes in all patients with the average admission and average follow-up data can be observed and evaluated.

\section{RESULTS}

To perform experiments on the upgraded SSD, 116 new patients belonging to the cervical degenerative diseases group, registered in the SSD database, were intensively examined. The changes in the Nurick and JOA scores during the preoperative and postoperative follow-up periods were evaluated using the SSD analytical evaluation module for 116 patients. Table I presents the gender and age distributions of these patients. Among the 116 patients, 30 were female (25.87\%). When the overall female distributions were analyzed, the mean value and standard deviation were calculated to be 54.46 and 9.77 , respectively. The remaining 86 patients were male $(74.13 \%)$, and the mean value and standard deviation of the male distributions were calculated to be 57.47 and 11.97, respectively.

Table II lists the results obtained from the values entered in the SSD application for the Nurick classification evaluation questionnaire during the preoperative and follow-up periods (6 months, 12 months). According to these values, the results of the improvements observed after the treatment process are presented in Table II. According to Table II, the mean values of the preoperative Nurick scale changed from 2.16 to 1.30 and 2.01 to 1.31 for females and males, respectively.

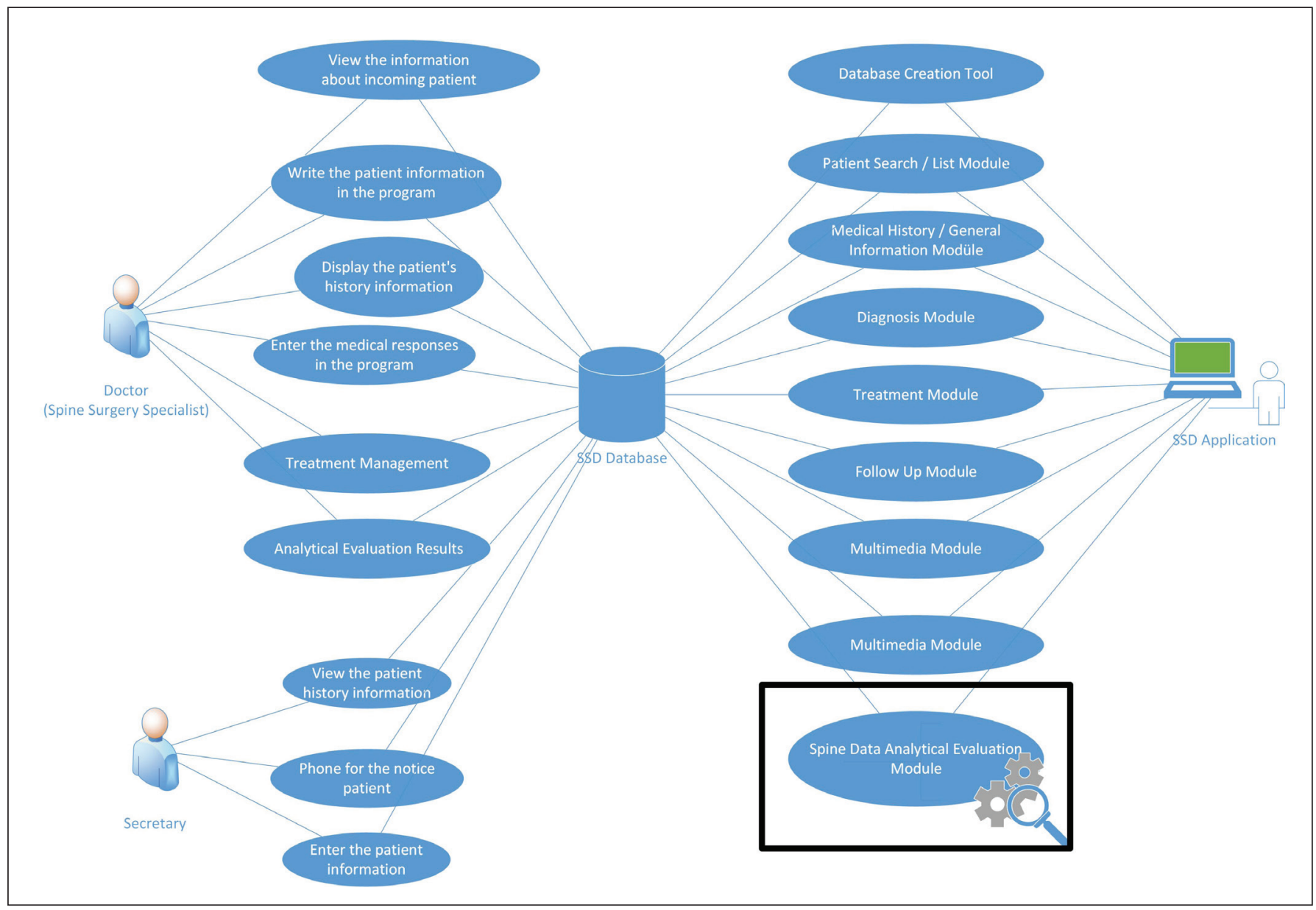

Figure 1: Use case diagram for the SSD. 

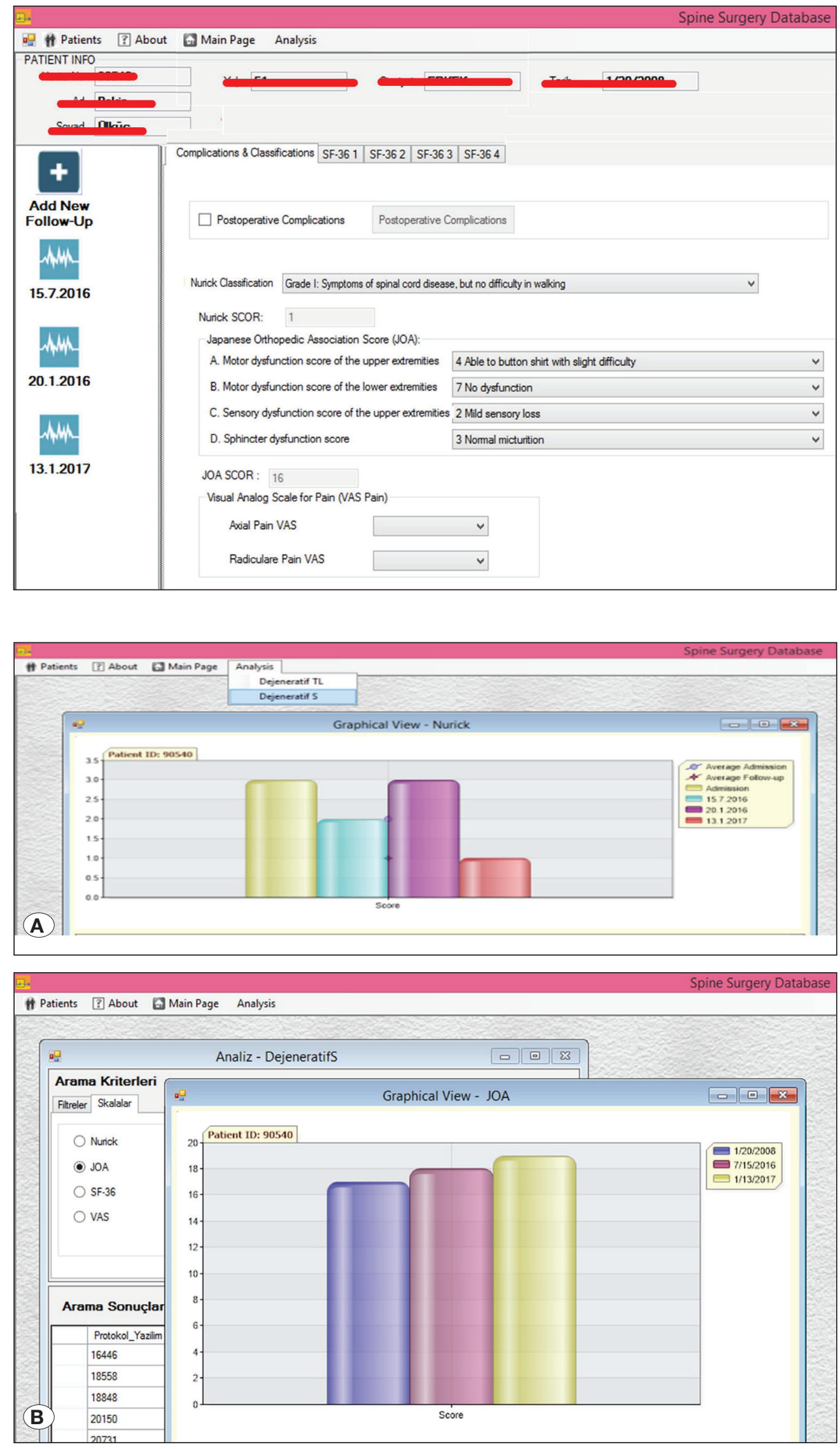

Figure 2: SSD follow-up module.

Figure 3: A) Graphical view Nurick, B) Graphical view - JOA in SSD. 


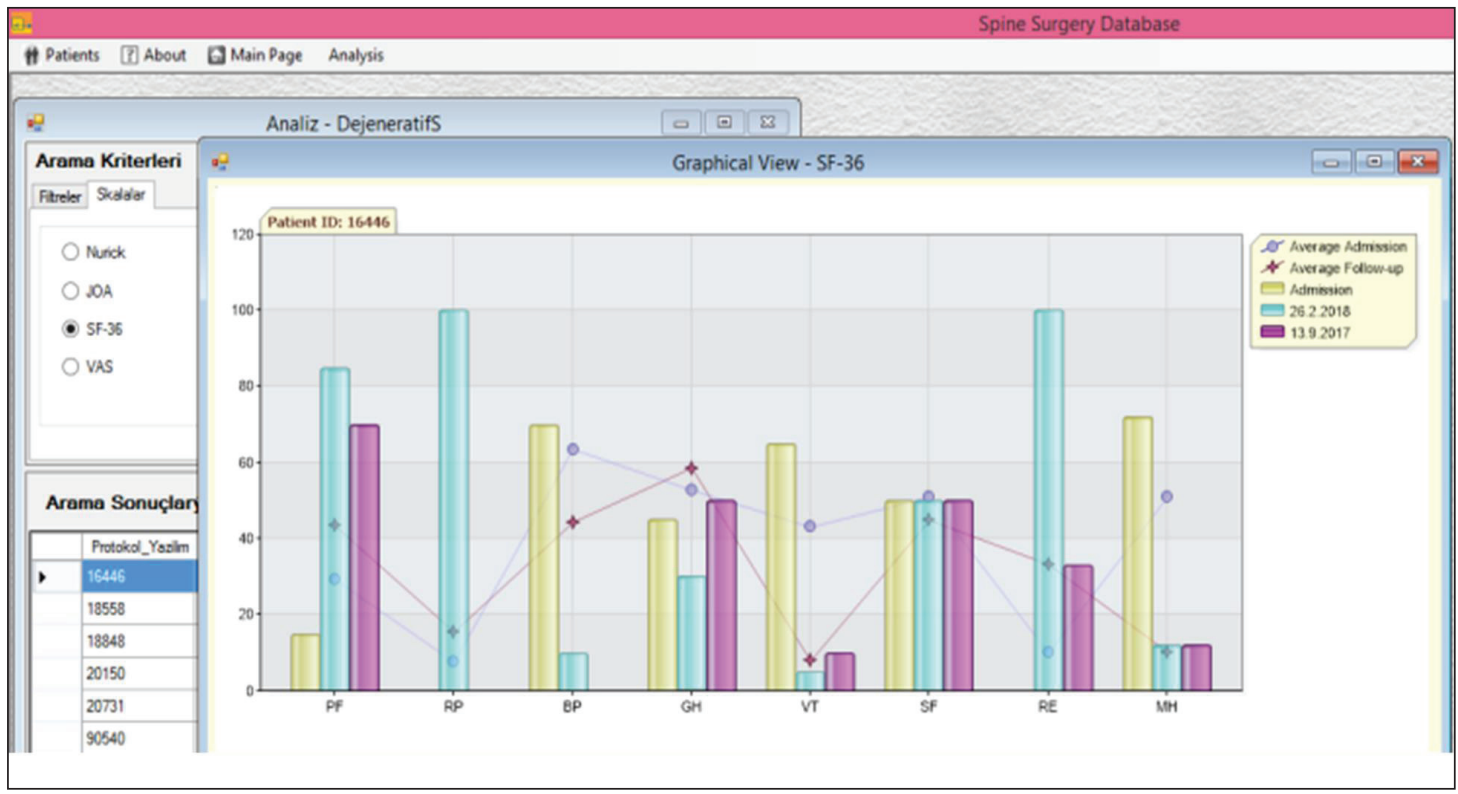

Figure 4: SF-36

analysis interface.

Table I: The Data Set of the Patients in "Degenerative Cervical Disorders" Distribution

\begin{tabular}{|c|c|c|c|c|c|c|c|}
\hline \multirow[t]{3}{*}{ Age } & \multicolumn{6}{|c|}{ Gender } & \multirow[t]{2}{*}{$\%$ distribution } \\
\hline & & Female & & & Male & & \\
\hline & $\mathbf{n}$ & Mean & Std.Dev. & $\mathbf{n}$ & Mean & Std.Dev. & \\
\hline$<30$ & - & & & 2 & 21.5 & 2.12 & 1.73 \\
\hline $30-40$ & 2 & 39 & 1.41 & 5 & 37.4 & 2.79 & 6.03 \\
\hline $41-50$ & 10 & 46.20 & 3.01 & 16 & 46.56 & 3.01 & 22.42 \\
\hline $51-60$ & 10 & 55.4 & 2.31 & 26 & 55.53 & 3.04 & 31.04 \\
\hline $61-70$ & 7 & 66.57 & 3.15 & 26 & 65.61 & 2.96 & 28.44 \\
\hline$>70$ & 1 & 74 & - & 11 & 74.36 & 3.23 & 10.34 \\
\hline
\end{tabular}

Table II: The Results of Pre-operative and Follow-Up Nurick Classification Evaluation

\begin{tabular}{lcccc}
\hline & \multicolumn{2}{c}{ Female $(\mathbf{n}=\mathbf{3 0})$} & \multicolumn{2}{c}{ Male $(\mathbf{n}=\mathbf{8 6})$} \\
\hline Mean & Std.Dev. & Mean & Std.Dev. \\
\hline Preoperative & 2.16 & 1.01 & 2.01 & 1.16 \\
\hline 6 months after surgery & 1.53 & 0.97 & 1.60 & 0.94 \\
\hline 12 months after surgery & 1.30 & 0.79 & 1.31 & 0.73 \\
\hline
\end{tabular}

Table III presents the JOA scores (motor dysfunction score of the upper and lower extremities) of 116 patients according to the data entered using the SSD follow-up module.

According to the results, the improvement status of a patient can be instantly viewed through the application in the SSD follow-up module. The obtained results show that the mean motor dysfunction scores of the upper extremities ranged from
3.6 to 4.1 and 3.5 to 4.12 in females and males, respectively. Similarly, the mean motor dysfunction scores of the lower extremities ranged from 4.83 to 5.86 and 4.82 to 5.87 in females and males, respectively.

The sensory dysfunction scores of the upper extremities and sphincter dysfunction scores that changed according to the JOA score are presented in Tables IV and V, respectively. In 
Table IV, the preoperative score is observed to be 1.93; it reached a value of 2.26 in the control group for females after 12 months. Additionally, the mean value changed from 2.02 to 2.24 in males. Table $\mathrm{V}$ presents that the preoperative score changed from 2.6 to 2.96 and 2.47 to 2.95 in females and males, respectively, in the follow-up after 12 months. The time performance of the patient data entry and evaluation (the main objective of this study) was tested; the 116 new patients of the cervical degenerative group were analyzed and evaluated using the SSD analytical module. First, the preoperative patient questionnaires (JOA and Nurick) were filled on paper (Experiment 1). Simultaneously, the same data were entered through the SSD GUI (SSD Experiment). The following day, the data on paper were transferred randomly to a statistical program (IBM SPSS version 24) (14) for Experiment 2. Finally, an evaluation procedure was performed in SPSS (Experiment 3). Each experiment was timed separately; Experiments 1, 2, and 3 were a part of the manual data entry process, whereas the SSD Experiment was a direct, paperless method. The time values obtained during the experiments are listed in Table VI.

Table III: The Results of Motor Dysfunction Score of the UPPER and LOWER Extremities

\begin{tabular}{|c|c|c|c|c|c|c|c|}
\hline & & Pre-operative & $\begin{array}{l}\text { Follow-Up } \\
6 \text { months }\end{array}$ & $\begin{array}{l}\text { Follow-Up } \\
12 \text { months }\end{array}$ & Pre-operative & $\begin{array}{l}\text { Follow-Up } \\
6 \text { months }\end{array}$ & $\begin{array}{l}\text { Follow-Up } \\
12 \text { months }\end{array}$ \\
\hline & & \multicolumn{3}{|c|}{$\begin{array}{l}\text { Motor dysfunction score of the } \\
\text { UPPER extremities }\end{array}$} & \multicolumn{3}{|c|}{$\begin{array}{l}\text { Motor dysfunction score of the } \\
\text { LOWER extremities }\end{array}$} \\
\hline Mean & \multirow{2}{*}{ Female } & 3.6 & 3.8 & 4.1 & 4.83 & 5.4 & 5.86 \\
\hline Std Dev. & & 0.85 & 0.84 & 0.66 & 1.46 & 1.24 & 1.1 \\
\hline Mean & \multirow{2}{*}{ Male } & 3.5 & 3.81 & 4.12 & 4.82 & 5.23 & 5.87 \\
\hline Std Dev. & & 0.89 & 0.84 & 0.62 & 1.3 & 1.18 & 1.02 \\
\hline
\end{tabular}

Table IV: The Results of Sensory Dysfunction Score of the Upper Extremities

\begin{tabular}{lcccc}
\hline & \multicolumn{2}{c}{ Female $(\mathbf{n}=\mathbf{3 0})$} & \multicolumn{2}{c}{ Male $(\mathbf{n}=\mathbf{8 6})$} \\
\hline Mean & Std.Dev. & Mean & Std.Dev. \\
\hline Preoperative & 1.93 & 0.52 & 2.02 & 0.4 \\
\hline months after surgery & 2.23 & 0.5 & 2.17 & 0.43 \\
\hline 12 months after surgery & 2.26 & 0.44 & 2.24 & 0.43 \\
\hline
\end{tabular}

Table V: The Results of Sphincter Dysfunction Score

\begin{tabular}{llccc}
\hline & \multicolumn{2}{c}{ Female $(\mathbf{n}=\mathbf{3 0})$} & \multicolumn{2}{c}{ Male $(\mathbf{n}=\mathbf{8 6})$} \\
\hline Mean & Std.Dev. & Mean & Std.Dev. \\
\hline Preoperative & 2.6 & 0.49 & 2.47 & 0.6 \\
\hline months after surgery & 2.9 & 0.4 & 2.87 & 0.36 \\
\hline 12 months after surgery & 2.96 & 0.18 & 2.95 & 0.21 \\
\hline
\end{tabular}

Table Vl: Experiments During Preoperative Data Entry Statistics

\begin{tabular}{|c|c|c|c|c|c|c|}
\hline & $\mathbf{n}$ & Sum & \multicolumn{2}{|c|}{ Mean } & Std.Dev. & Variance \\
\hline Experiment 1 & 116 & $319.7 \mathrm{~min}$. & $2.75 \mathrm{~min}$. & 0.08 & 0.43 & 1.90 \\
\hline Experiment 2 & 116 & $548.6 \mathrm{~min}$. & $4.71 \mathrm{~min}$. & 0.17 & 0.86 & 7.42 \\
\hline Experiment 3 & 116 & $4.6 \mathrm{~min}$. & $0.04 \mathrm{~min}$ & & & \\
\hline
\end{tabular}

SSD Experiment: Spine Surgery Database Experiment 
Table VI presents that the manual questionnaire method (the sum of Experiments 1, 2, and 3) required $7.5 \mathrm{~min}(2.75+4.71$ $+0.04)$ per patient on average. The same input and evaluation process took 4.06 min per patient on average when the newly upgraded SSD module was used. Overall, considering the total time required for 116 patients, the upgraded SSD method performs data entry and analysis in $471.9 \mathrm{~min}$, whereas the manual method requires a staggering $872.9(319.7+548.6+$ 4.6) min. Thus, a total of $401 \mathrm{~min}$, on average, can be gained while entering the data for 116 patients when the SSD module is used. The results indicate an improvement of $45.64 \%$ in time efficiency, ignoring the incorrect entries or transfer of data during the paper-based manual method.

\section{DISCUSSION}

With the increased use of paperless methods in hospitals, medical data are being generally converted to digital records. In addition, it has become necessary to develop custom software with different tools to meet the requirements of specialists. The extraction of semantic consequences of data from the stored records is becoming increasingly important. In this study, an SSD analytical evaluation module was presented and combined with the SSD spine surgery software. The SSD software has been used by spine surgeons since 2006 . The upgraded module facilitated the development of a userfriendly interface that produces instant, accurate, and sharable results. The experimental results demonstrate that paperless methods produce more time-efficient results because they eliminate duplicated data entries. Additionally, these methods help in eliminating the manual errors stated in (1).

Spine Tango is another specialized software used in the field of spine surgery. Borcek et al. attempted to store the patient follow-up data using their customized Spine Tango software in the Gazi University Neurosurgery Department (4). However, the Spine Tango database was web-based and encountered security problems related to the storage and logging of data. Therefore, specific applications and platforms such as SSD are required to store and manage the follow-up data in a secure manner.

\section{CONCLUSION}

A spine surgery database application, SSD, developed in 2006, was recently upgraded with an analytical evaluation module. The SSD has become a specialized tool that assists physicians in the processes of data entry and post-surgery evaluation. Thus, the upgraded SSD established an integrated electronic health record infrastructure environment for spine surgeons that is free of human errors. In this study, the changes observed in the Nurick and JOA scores of cervical degenerative patients, recorded in the SSD, were analyzed. Experiments with 116 new patients demonstrated that a surgeon can gain $3.44 \mathrm{~min}$ per patient by using the proposed SSD. The overall gain in efficiency for 116 patients was found to be $45.64 \%$. Thus, it is evident that a paperless patient tracking environment with customized software can provide experts with analytical solutions for the efficient evaluation and tracking of data.

\section{REFERENCES}

1. Ahmadi $\mathrm{H}$, Nilashi $\mathrm{M}$, Ibrahim $\mathrm{O}$, Ramayah $\mathrm{T}$, Wai $\mathrm{M}$, Alizadeh M: Exploring potential factors in total hospital information system adoption. Journal of Soft Computing and Decision Support Systems 2(1):52-59, 2015

2. Ahmadi H, Nilashi M, Shahmoradi L, Ibrahim O: Hospital information system adoption: Expert perspectives on an adoption framework for Malaysian public hospitals. Computers in Human Behavior 67:161-189, 2017

3. Benzel EC, Lancon J, Kesterson L, Hadden T: Cervical laminectomy and dentate ligament section for cervical spondylotic myelopathy. Journal of Spinal Disorders 4(3):286295, 1991

4. Borcek AO, Bulduk EB, Civi S, Emmez H, Kaymaz M: Spine Tango in Turkish: Development of a local registry system. Turk Neurosurg 27(2):237-244, 2017

5. Brazier JE, Harper R, Jones NM, O'Cathain A, Thomas KJ, Usherwood T, Westlake L: Validating the SF-36 health survey questionnaire: new outcome measure for primary care. BMJ 305(6846):160-164, 1992

6. Hogaboam L, Ragel B, Daim T: Development of a Hierarchical Decision Model (HDM) for Health Technology Assessment (HTA) to design and implement a new patient care database for low back pain. Kanazawa: 2014 Portland International Conference on Management of Engineering \& Technology (PICMET), 2014:3511-3517

7. Keller A, Von Ammon K, Klaiber R, Waespe W: Spondylogenic cervical myelopathy: Conservative and surgical therapy. Schweizerische Medizinische Wochenschrift 123(36):1682-1691, 1993

8. Kudyba SP: Healthcare Informatics: Improving Efficiency and Productivity. CRC Press, 2010

9. McHorney CA, Ware JE, Raczek AE: The MOS 36-item shortform health survey (Sf-36): II. Psychometric and clinical tests of validity in measuring physical and mental health constructs. Medical Care 31(3):247-263, 1993

10. Metaxiotis K: Encyclopedia of Knowledge Management. In: Healthcare Knowledge Management, 2011:366-375

11. Raghupathi W, Raghupathi V: Big data analytics in healthcare: Promise and potential. Health Inf Sci Syst 2(1):3, 2014

12. Utku S, Baysal H, Zileli M: Spine surgery database: A Turkish registry for spinal disorders. Turk Neurosurg 20(2):223-230, 2010

13. Visualization utility. netcharting. http://www.dotnetcharting. com/. Accessed September 15, 2019

14. www.ibm.com/productsspss-statistics/. IBM SPSS Statistics. https://www.ibm.com/products/spss-statistics. Accessed September 20, 2019

15. Yilmaz A, Altug F, Coskun E: Pain, disability status and psychological factors in patients with chronic low back pain. Turkiye Klinikleri Journal of Medical Sciences 32(5):12781283, 2012

16. Yonenobu K, Abumi K, Nagata K, Taketomi E, Ueyama K: Interobserver and intraobserver reliability of the Japanese Orthopaedic Association scoring system for evaluation of cervical compression myelopathy. Spine 26(17):1890-1895, 2001 